\title{
Principes de préparation et de congélation des spermatozoïdes testiculaires humains
}

\author{
Geneviève GRIZARD, André FORCE, Laurent JANNY, Daniel BOUCHER \\ Laboratoire de Biologie du Développement et de la Reproduction, \\ CECOS, Clermont-Ferrand
}

\section{RESUME}

L'intérêt et la faisabilité de la cryoconservation des spermatozoïdes testiculaires humains sont maintenant bien démontrés. Toutefois, la réalisation de la congélation de ces spermatozoïdes reste empirique, elle est basée essentiellement sur les techniques utilisées pour les spermatozoïdes de l'éjaculât. Les spermatozoïdes testiculaires sont considérés comme des gamètes encore «immatures» et sont toujours retrouvés en quantité limitée. Une meilleure connaissance de leurs caractéristiques cryobiologiques contribuerait à mieux définir le type d'échantillon à congeler (tissu, suspension cellulaire contenant des spermatozoïdes....), à faire évoluer les processus de congélation/décongélation et la nature des milieux cryoprotecteurs et à adapter les conditions de stockage. Compte tenu des caractéristiques fonctionnelles de ces spermatozoïdes et du fait qu'ils sont utilisés exclusivement en ICSI, de nouveaux tests d'évaluation de la résistance à la congélation basés notamment sur leur qualité nucléaire devraient être développés.

Mots clés : congélation, décongélation, spermatozoïdes testiculaires, homme

\section{INTRODUCTION}

La cryoconservation des spermatozoïdes de l'éjaculât est devenue une pratique médicale courante. Plus récemment, avec le développement de la technique de fécondation in vitro avec microinjection dans le cytoplasme ovocytaire (ICSI), la cryoconservation a été appliquée aux spermatozoïdes testiculaires avec succès puisque de nombreuses équipes rapportent des grossesses obtenues par ICSI avec des spermatozoïdes testiculaires cryoconservés de patients présentant une azoospermie excrétoire ou sécrétoire.

En réalisant de façon asynchrone le prélèvement testiculaire et la tentative d'ICSI, une stimulation et une ponction d'ovocytes pourront être évitées chez des patients pour lesquels aucun spermatozoïde n'est retrouvé dans la biopsie du conjoint. En outre, la programmation des interventions au niveau des équipes médico-chirurgicales est facilitée et le biologiste peut disposer d'un temps supplémentaire pour une recherche minutieuse et un traitement éventuel (culture in vitro) des spermatozoïdes susceptible d'améliorer leur mobilité. De plus, à partir d'un seul prélèvement chirurgical, la quantité de spermatozoïdes cryoconservés peut permettre de réaliser plusieurs ICSI évitant de nouvelles interventions chirurgicales pouvant augmenter les risques de complications notamment chez des patients présentant une azoospermie sécrétoire et pour lesquels la taille du testicule est réduite [26].

Pour certains auteurs, les ICSI réalisées avec des spermatozoïdes testiculaires cryoconservés conduisent à des taux de fécondation, de clivage et d'implantation embryonnai-

\section{Correspondance :}

Dr Geneviève GRIZARD - Laboratoire de Biologie du Développement et de la Reproduction - CECOS, Hôtel-Dieu, Boulevard Léon Malfreyt, 63058 Clermont-Ferrand -

Tel 04.73.750.230 - Fax 04.73.750.219 -

Email ggrizard@chu-clermontferrand.fr 
res ou de naissances plus faibles que ceux obtenus avec des spermatozoïdes testiculaires frais [4, 9, 33], alors que pour d'autres, il n'existerait aucune différence $[7,32]$.

Comparés aux spermatozoïdes éjaculés, les spermatozoïdes testiculaires sont encore considérés comme des gamètes «immatures». En effet, au cours du transit épididymaire, se produisent des remaniements morphologiques, biochimiques ou fonctionnels importants. Par exemple, il existe une augmentation de la fluidité membranaire [12] et une modification du rapport volume/surface membranaire [1], deux paramètres critiques en cryobiologie et qui devraient être pris en compte dans l'établissement des protocoles de congélation $\lceil 10,20]$. Cependant, compte tenu des très faibles quantités de spermatozoïdes disponibles, aucune étude spécifique concernant les caractéristiques cryobiologiques des spermatozoïdes testiculaires humains n'a été réalisée et les protocoles de cryoconservation de ces spermatozoïdes ont été adaptés à partir de ceux utilisés pour les spermatozoïdes de l'éjaculât.

\section{RECUEIL ET TRAITEMENT DES PRELEVEMENTS TESTICULAIRES AVANT LA CONGEI.ATION}

Deux techniques chirurgicales de prélèvements testiculaires sont actuellement utilisées, la biopsie chirurgicale à ciel ouvert et la ponction percutanée à l'aiguille $[3,17,18,22]$. Les prélèvements sont alors rapidement immergés dans un milieu de survie avant d'être transportés au laboratoire, généralement à température ambiante $\left(20-25^{\circ} \mathrm{C}\right)$ pour être traités.

La revue de la littérature $[2,16,19,27]$ montre que la nature des prélèvements cryoconservés est très hétérogène : fragments de tissus intacts, tubes séminifères à partir desquels les spermatozoïdes peuvent être plus facilement extraits après décongélation, suspensions cellulaires plus ou moins purifiées, ou spermatozoïdes isolés. A partir des prélèvements chirurgicaux, les spermatozoïdes sont extraits soit par un traitement mćcanique basé sur une technique manuelle permettant une dilacération des tubes séminifères, soit par une digestion enzymatique à l'aide de collagénase (collagénase de type IA ou de type IV). Les suspensions cellulaires obtenues contenant les spermatozoïdes peuvent être congelées directement après dilution avec le milieu cryoprotecteur, ou subir un traitement (migration sur gradient de densité) permettant d'isoler les spermatozoïdes avant la congélation. Une culture in vitro des préparations obtenues peut être aussi réalisée avant congélation pour éventuellement initier une mobilité des spermatozoïdes [28].

Des naissances ont été rapportées, quelle que soit la nature du prélèvement cryoconservé, cependant il existe peu d'études systématiques comparant les résultats obtenus avec ces différents types de prélèvements. Crabbe et al. [2] ont réalisé une étude sur 14 biopsies. Pour chaque échantillon. deux protocoles ont été testés : congélation d'un fragment de tissu et dilacération après décongélation ou dilacération du tissu frais puis congélation et décongélation d'une suspension hétérogène. La congélation après dilacération des cellules testiculaires permet d'obtenir une quantité plus importante de spermatozoïdes vivants et mobiles que la congélation du tissu.

\section{LES DIFFERENTES ETAPES DE LA CRYOCONSERVATION DES SPERMATO- ZOÏDES}

La cryoconservation comporte quatre grandes étapes : la mise en contact des spermatozoïdes avec le milieu cryoprotecteur suivie du refroidissement et de la congélation, puis le stockage à $-196^{\circ} \mathrm{C}$ et finalement la décongélation et le retrait du cryoprotecteur.

Ce processus entraînc d'importantes variations du volume du spermatozoïde liées aux flux d'eau et de cryoprotecteur entre les milieux intra et extracellulaires, consécutifs aux modifications d'osmolalité provoquées par l'addition du cryoprotecteur (hypertonique) et à la formation des cristaux de glace (Figure 1). En effet, au fur et à mesure que l'eau extracellulaire se congèle, les solutés s'accumulent dans l'eau liquide. Il y a donc une augmentation du gradient osmotique entre le milieu intracellulaire et le milieu extracellulaire provoquant une sortie de l'eau et une déshydratation de la cellule ; c'est donc sous un volume réduit que le spermatozoïde sera conservé. Inversement, au moment de la décongélation, la diminution de la concentration des solutés dans le milieu extracellulaire entraîne une entrée d'eau dans le spermatozoïde qui va alors transitoirement «gonfler», puis une sortie du cryoprotecteur et finalement le spermatozoïde devrait retrouver son volume initial $[1,14]$.

\section{CONSEQUENCES DE LA CRYOCONSER- VATION SUR LES SPERMATOZOÏDES}

De nombreux travaux ont rapporté des effets délétères de la congélation/décongélation sur les spermatozoïdes de l'éjaculât, que ce soit au niveau morphologique, membranaire ou nucléaire et se traduisant par des altérations fonctionnelles (baisse de mobilité, du pouvoir fécondant) [24]. Par contre, les conséquences de la cryoconservation sur les spermatozoïdes testiculaires ont été peu étudiées. Nogueira et al. [23] ont analysé en microscopie optique et électronique les spermatozoïdes après congélation/décongélation de biopsies testiculaires de sujets porteurs d'une azoospermie excrétoire. Ils ont observé des lésions au niveau des membranes plasmiques et nucléaires (gonflement, fragmentation) de la région post-acrosomique et de l'acrosome 


\section{$\Rightarrow$ cryoprotecteur \\ $\Rightarrow$ eau}

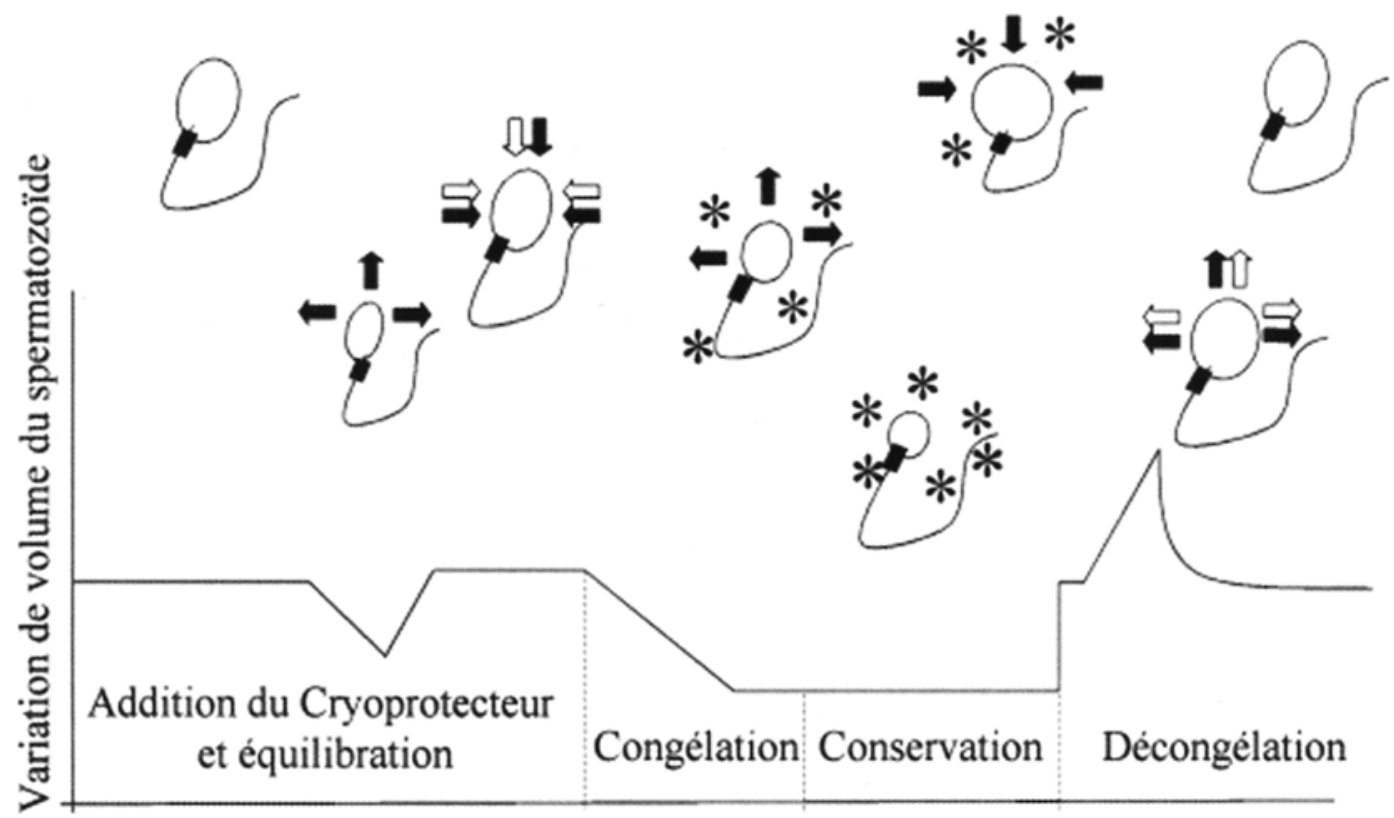

Figure 1 : Variation du volume du spermatozoïde au cours du processus de congélation/décongélation. Influence des flux du cryoprotecteur et de l'eau entre le milieu intracellulaire et le milieu extracellulaire. D'après Hammerstedt et al. [14].

(formation de vacuoles, expulsion du contenu acrosomique). Ces altérations sont assez comparables à celles observées après décongélation de spermatozoïdes éjaculés. Toutefois, les conséquences sur la fécondation et le développement des embryons obtenus en ICSI (technique obligatoire pour des spermatozoïdes testiculaires) restent à préciser.

Des études réalisées chez des sujets fertiles (témoins) montrent que la fréquence des spermatozoïdes dont l'ADN est intact (apprécié par la technique COMET) est significativement plus élevée pour les spermatozoïdes testiculaires que pour les spermatozoïdes éjaculés. En outre, pour les spermatozoïdes testiculaires provenant de sujets fertiles ou azoospermiques, la congélation/décongélation des spermatozoïdes sous forme de tissu ou de suspensions cellulaires, n'entraîne aucune altération supplémentaire de l'ADN [30].

La faible quantité de spermatozoïdes testiculaires disponible, limite les tests permettant d'évaluer leur résistance à la cryoconservation. Ceux-ci reposent essentiellement sur l'analyse de la vitalité et de la mobilité post-décongélation et sur les taux de fécondation, de clivage et d'implantation embryonnaires, de grossesses et de naissances. Cependant, pour optimaliser les processus des cryoconservations et compte tenu des caractéristiques spécifiques des spermatozoïdes testiculaires et de leur utilisation (ICSI), il serait souhaitable de développer d'autres tests permettant d'apprécier des altérations plus discrètes.

\section{MILIEUX DE CONGELATION ET PROTO- COLE DE CONGELATION / DECONGELATION}

Aucune étude spécifique sur l'efficacité des milieux de congélation n'a été réalisée pour les spermatozoïdes testiculaires et les milieux utilisés sont ceux habituellement retenus pour les spermatozoïdes éjaculés, contenant le glycérol comme cryoprotecteur. Dans le sperme, nous avons montré que le liquide séminal a un effet protecteur sur les spermatozoïdes au cours du processus de congélation/décongélation qui s'expliquerait notamment par son pouvoir anti-oxydant $[11,25]$. Pour les spermatozoïdes testiculaires, l'addition dans les milieux cryoprotecteurs d'anti-oxydants pour pallier à l'absence de liquide séminal pourrait donc s'avérer bénéfique.

Dans le cas des spermatozoïdes éjaculés, l'action de différentes substances additionnées au milieu cryoprotecteur a été testée. C'est le cas de la pentoxifylline. Pour certains auteurs $[6,15]$, elle aurait un effet bénéfique au cours du processus de congélation / décongélation sur la mobilité et sur l'acrosome, alors que pour d'autres, elle entraînerait une augmentation de la mobilité seulement si elle est ajoutée au milieu après décongélation [29]. Le mécanisme d'action de la pentoxifylline est complexe puisque, en tant qu'inhibiteur de la phosphodiestérase, elle permet de maintenir le taux intracellulaire d'AMPc à un certain niveau et d'autre part, en se comportant comme un piégeur de radi- 
caux oxygénés, elle limiterait les effets délétères du stress oxydant. La supplémentation des milieux par la pentoxifylline pourrait donc aussi contribuer à améliorer la tolérance à la congélation des spermatozoïdes testiculaires.

La vitesse de congélation est un paramètre important puisqu'elle conditionne la vitesse de formation et la taille des cristaux de glace, leur présence éventuelle à l'intérieur de la cellule.

Si la vitesse de refroidissement est lente, des cristaux de glace se forment autour du spermatozoïde, puis grandissent, et le spermatozoïde se déshydrate progressivement et se trouve exposé à de forte concentration de soluté, puis finalement meurt. Inversement, si la vitesse de refroidissement est très rapide, le flux d'eau extra-cellulaire est très faible, des cristaux de glace se forment alors à l'intérieur du spermatozoïde et lorsqu'ils atteignent une certaine taille, provoquent des lésions et la mort de la cellule [1]. Il existe donc une zone optimum de vitesse de refroidissement pour laquelle les lésions seront minimum et donc la survie maximum [5].

D'après la littérature, les spermatozoïdes testiculaires sont congelés soit rapidement en une seule étape au cours de laquelle, les échantillons sont maintenus 8 à 30 minutes dans les vapeurs d'azote avant d'être plongés dans l'azote liquide, soit progressivement et de façon contrôlée avec des vitesses de refroidissement croissantes. Le programme que nous utilisons, comporte trois paliers de refroidissement : $5^{\circ} \mathrm{C} /$ minute entre la température ambiante et $-4^{\circ} \mathrm{C}$; $10^{\circ} \mathrm{C} /$ minute jusqu'à $-30^{\circ} \mathrm{C}, 20^{\circ} \mathrm{C} /$ minute jusqu'à $-140^{\circ} \mathrm{C}$ et finalement les paillettes sont plongées et stockées dans l'azote liquide. Aucune étude comparative de ces deux méthodes n'a été réalisće pour les spermatozoïdes testiculaires. Toutefois, en se référant aux résultats obtenus à partir de travaux réalisés sur des spermes altérés, une congélation progressive et contrôlée donne de meilleurs résultats qu'une congélation réalisée dans les vapeurs d'azote [13, 31] en termes de vitalité, mobilité, morphologie et condensation de la chromatine. De plus, il est recommandé d'associer une décongélation à $22^{\circ} \mathrm{C}$ à une congélation lente et progressive et une décongélation à $37^{\circ} \mathrm{C}$ dans le cas d'une congélation rapide [31]. L'addition du cryoprotecteur à la suspension de spermatozoïdes, avant congélation et son retrait après décongélation, doivent se faire de façon progressive et contrôlée pour éviter les chocs osmotiques [8].

D'après une étude récente [21], la vitrification qui se caractérise par un refroidissement très rapide (environ 10 $000^{\circ} \mathrm{C} /$ minute) et l'absence de cryoprotecteur, offre des perspectives intéressantes pour la cryoconservation des spermatozoïdes humains. Des spermatozoïdes éjaculés humains sélectionnés par migration ascendante et soumis à une vitrification, présentent, après décongélation, des caractéristiques (vitalité, morphologie, mobilité) compara- bles à celles de spermatozoïdes cryoconservés selon un protocole classique, et des embryons ont été obtenus en fécondation in vitro avec ces spermatozoïdes.

\section{CONCLUSION}

Si l'intérêt de la cryoconservation des spermatozoïdes testiculaires est maintenant bien admis, les techniques utilisées restent encore empiriques. Des altérations, qui restent encore mal identifiées, du spermatozoïde peuvent se produire à toutes les étapes : traitement des tissus, mise en contact avec le milieu cryoprotecteur, processus de congélation/décongélation.

Une meilleure connaissance des caractéristiques morphologiques, fonctionnelles et cryobiologiques des spermatozoïdes testiculaires devrait contribuer à faire évoluer ces techniques et à optimaliser les procédures.

\section{REFERENCES}

1. AGCA Y., CRITSER J.K. : Cryopreservation of spermatozoa in assisted reproduction. Sem. Reprod. Med., 2002, 20 : 15-23.

2. CRABBE E., VERHEYLN G., TOURNAYE H. et al. : Freezing of testicular tissue as a minced suspension preserves sperm quality better than whole biopsy freezing when glycerol is used as cryoprotectant. Int. J. Androl., 1999, $22: 43-48$.

3. CRAFT 1., TSIRIGOTIS M. : Simplified recovery, preparation and cryopreservation of testicular spermatozoa. Hum. Reprod., 1995, $10: 1623-1627$.

4. DE CROO I., VAN DER ELST J., EVERAERT K. et al. : Fertilization, pregnancy and embryo implantation rates after ICSI with fresh or frozen-thawed testicular spermatozoa. Hum. Reprod., 1998, $13: 1893-1897$.

5. DEVIREDDY R.V., SWANLUND D.J., ROBERTS K.P. et al. : The effect of extracellular ice and cryoprotective agents on the water permeability parameters of human sperm plasma membrane during freezing. Hum. Reprod., 2000, 15 : 1125-1135.

6. ESTEVES S.C, SHARMA R.K., THOMAS A.J. et al. : Cryopreservation of human spermatozoa with pentoxifylline improves the post-thaw agonist-induced acrosome reaction rate. Hum. Reprod., 1998, $13: 3384-3389$.

7. FRIEDLER S., RAZIEL A., STRASSBURGER D. et al. : Outcome of ICSI using fresh and cryopreserved-thawed testicular spermatozoa in patients with non-mosaic Klinefelter's syndrome. Hum. Reprod., 2001, $16: 2616-2620$.

8. GAO D.Y., LIU J., LIU C. et al. : Prevention of osmotic injury to human spermatozoa during addition and removal of glycerol. Hum. Reprod., 1995, $10: 1109-1122$.

9. GIL-SALOM M., ROMERO J., MINGUEZ Y. et al. : Pregnancies after intracytoplasmic sperm injection with cryopreserved testicular spermatozoa. Hum. Reprod., 1996, $11:$ 1309-1313.

10. GIRAUD M.N., GRIZARD G., BOUCHER D. : Membrane fluidity predicts the outcome of cryopreservation of human spermatozoa. Hum. Reprod., 2000, $15: 2160-2164$.

11. GRIZARD G., CHEVALIER V., GRIVEAU J.F. et al. : Influence 
of seminal plasma on cryopreservation of human spermatozoa in a biological material-free medium : study of normal and lowquality semen. Int. J. Androl., 1999, 22 : 190-196.

12. HAIDL G., OPPER C. : Changes in lipids and membrane anisotropy in human spermatozoa during epididymal maturation. Hum. Reprod., 1997, $12: 2720-2723$.

13. HAMMADEH M.E., DEHN C.H., HIPPACH M. et al. : Comparison between computerized slow-stage and static liquid nitrogen vapour freezing methods with respect to the deleterious effect on chromatin and morphology of spermatozoa from fertile and subfertile men. Int. J. Androl., 2001, 24 : 66-72.

14. HAMMERSTEDT R.H., GRAHAM J.K., NOLAN J.P. : Cryopreservation of mammalian sperm : what we ask them to survive. J. Androl., 1990, 11 : 73-88.

15. HAMMITT D.G., BEDIA. E., ROGERS P.R. et al. : Comparison of motility stimulants for cryopreserved human semen. Fertil. Steril., 1989, 52 : 495-502.

16. KÜPFER W., AL-HASANI S., JOHANNISSON R, et al. : The use of cryopreserved mature and immature testicular spermatozoa for intracytoplasmic sperm injection : risks and limitations. Sem. Reprod. Med., 2002, $20: 25-35$.

17. LE LANNOU D., GRIVEAU J.F., LAURENT M.C. et al. : Azoospermie et microinjection. Andrologie, 2001, 11 : 142-148.

18. LORNAGE J., GISCARD D'ESTAING S., LEJEUNE H. et al. : Les prélèvements testiculaires dans les azoospermies sécrétoires: le point de vue du biologiste de la reproduction sur la lecture du prélèvement. Andrologie, 2001, $11:$ 127-132.

19. MARMAR J. : The emergence of specialized procedures for the acquisition, processing and cryopreservation of epididymal and testicular sperm in connection with intracytoplasmic sperm injection. J. Androl., 1998, $19: 517-526$.

20. MORRIS C.J. : A new development in the cryopreservation of sperm. Hum. Fertil., 2002, 5 : 23-29.

21. NAWROTH F., ISACHENKO V., DESSOLE S. et al. : Vitrification of human spermatozoa without cryoprotectants. Cryo Letters, $2002,23: 93-102$.

22. NIJS M., VAN DER ELST J. : Biological aspects of testicular sperm extraction. Eur. Obstet. Gynecol. Reprod. Biol., 2000, 92 : 1-6.

23. NOGUEIRA D., BOURGAIN C., VERHEYEN G. et al. : Light and electron microscopic analysis of human testicular spermatozoa and spermatids from frozen and thawed testicular biopsies. Hum. Reprod., 1999, 14 : 2041-2049.

24. ROYERE D., BARTHELEMY C., HAMAMAH S. et al. : Cryopreservation of spermatozoa : a 1996 review. Hum. Reprod. Update, 1996, 2 : 553-559.

25. SAEZ F., MOTTA C., BOUCHER D. et al. : Antioxydant capacity of prostasomes in human semen. Mol. Hum. Reprod., 1998, 4: 667-672.

26. SCHLEGEL P.N. : Testicular sperm extraction : micro-dissection improves sperm yield with minimal tissue excision. Hum. Reprod., 1999, 14 : 131-135.

27. SCHLEGEL P.N., PALERMO G.D., GOLDSTEIN M. et al : Testicular sperm extraction with intracytoplasmic sperm injec- tion for non-obstructive azoospermie. Urology, 1997, 49 : 435440 .

28. SCHUBERT B., GRIZARD G., BOUCHER D. : Utilisation des spermatozoïdes testiculaires en ICSI. Intérêt de la culture in vitro. Andrologie, 2001, $11: 9-20$.

29. STANIC P., SONECKI Z., SUCHANEK E. : Effect of pentoxifylline on motility and membrane integrity of cryopreserved human spermatozoa. Int. J. Androl., 2002, 25 : 186-190.

30. STEELE E.K., Mc CLURE N., LEWIS S. : Comparison of the effects of two methods of cryopreservation on testicular sperm DNA. Fertil. Steril., 2000, $74:$ 450-453.

31. VERHEYEN G., PLETINCX I., VAN STEIRTEGHEM A. : Effect of freezing method, thawing temperature and post-thaw dilution/washing on motility (CASA) and morphology characteristics of high quality human sperm. Hum. Reprod., 1993, 8 : 1678-1684.

32. WINDT M.L., COETZEE K., KRUGER T.F. et al. : Intracytoplasmic sperm injection with testicular spermatozoa in men with azoospermia. J. Assist. Reprod. Genet, 2002, 19 : 53-59.

33. WOOD S., THOMAS K., SCHNAUFFER K. et al, : Reproductive potential of fresh and cryopreserved epididymal and testicular spermatozoa in consecutive intracytoplasmic sperm injection cycles in the same patients. Fertil. Steril., 2002, $77: 1162-1166$.

\section{ABSTRACT}

\section{Principles of testicular sperm preparation and cryo- preservation}

\section{Geneviève GRIZARD, André FORCE, Laurent JANNY, Daniel BOUCHER}

The advantages and feasibility of human testicular spermatozoa cryoconservation for intracytoplasmic sperm injection (ICSI) have now been clearly demonstrated. However, the freezing protocol is based on empirical knowledge obtained from freezing of ejaculated spermatozoa. Testicular spermatozoa may not be fully mature gametes and may also be retrieved in only limited quantities. Little research has been conducted to determine whether they have the same cryobiological requirements as ejaculated spermatozoa. A better understanding of their cryobiological features and assessment of possible subcellular changes after thawing would help to optimize testicular preparations for cryopreservation (whole biopsies, seminiferous tubules, 
shredded suspension, single spermatozoa, etc.), freezing-thawing procedure, freezing media, and storage. Finally, there is a growing need for welldefined criteria (nuclear quality, etc.) to evaluate the tolerance of testicular spermatozoa to freezing-thawing procedure for ICSI

Key-Words: freezing, thawing, testicular spermatozoa, man 